\title{
An HD-Zip IV transcription factor protein NbGL3 regulates glandular trichome initiation in tobacco
}

\author{
Y.S. TIAN ${ }^{1,2}$, T.W. DAI ${ }^{2}$, C.X. FAN ${ }^{2}$, J. ZHOU ${ }^{1}$, X.L. REN ${ }^{1 *}$, and Y. XU ${ }^{2 *}$ \\ Molecular Genetics Key Laboratory of China Tobacco, Guizhou Academy of Tobacco Science, \\ Guiyang 550081, P.R. China ${ }^{1}$ \\ College of Life Sciences, Sichuan University, Chengdu 610065, P.R. China ${ }^{2}$
}

\begin{abstract}
Glandular trichomes, a specialized multicellular structure, are considered as biofactories due to their capability to synthesize and secrete a large amount of secondary metabolites. Tobacco leaves have a high density of glandular trichomes that produce huge amounts of secondary metabolites, which can be used as important industrial raw materials. However, molecular mechanism controling glandular trichome development in tobacco still remains largely unknown. In the present study, we found that $N b G L 3$, an HD-Zip IV family gene from Nicotiana benthamiana, was highly expressed in mature leaves, and ethylene or auxin application could increase the expression of $N b G L 3$. Virus induced gene silencing $N b G L 3$ resulted in a decreased glandular trichome density on leaves. Putative downstream genes, such as micro trichome, cyclin $B 2$, and wax inducer 1 like were down regulated in $N b G L 3$ silenced plants. Our results demonstrate that $N b G L 3$ could positively regulate initiation of glandular trichomes in tobacco.
\end{abstract}

Additional key words: auxin, ethylene, Nicotiana benthamiana, secondary metabolites, virus induced gene silencing.

\section{Introduction}

Plant trichomes, the epidermal outgrowths covering most aerial plant tissue surfaces, are found in a very large number of plant species. According to their morphology, trichomes could be classified into glandular or nonglandular, unicellular or multicellular, branched or nonbranched (Werker 2000, Pattanaik et al. 2014). Trichomes play various roles in plant growth and development, including protection of plant from UV radiation, arthropod herbivores, and pathogens (Kang et al. 2016), and from excess transpiration (Kang et al. 2014). Glandular trichomes are characterized by the ability of head cells to secrete or store large quantities of secondary metabolites (Huchelmann et al. 2017).

Tobacco shoots are covered with a high density of glandular trichomes. Tobacco glandular trichome cells secret and store huge amounts of terpenes, alkanes, acyl sugars, nicotine, fatty acid derivatives, and flavonoids (Roda et al. 2003), together representing up to $16 \%$ of the dry mass of the leaf (George 1991, Wagner et al. 2004). These exudates are important for aromas, flavours, and pharmaceuticals (Schilmiller et al. 2010, Huchelmann et al. 2017). Molecular mechanisms controlling trichome development in model plant Arabidopsis have been extensively studied and reviewed (Serna and Cathie 2006, Pesch and Hülskamp 2009, Pattanaik et al. 2014, Yan et al. 2014). However, previous study indicated that transcription networks controlling trichome development are different between tobacco and Arabidopsis. Overexpression of myeloblastosis related transcription factor (MIXTA) from Antirrhinum majus and $W^{V}$ gene from tomato could promote glandular trichome initiation in tobacco (Payne et al. 1999, Yang et al. 2015). Most recent reports demonstrated that $\mathrm{C} 2 \mathrm{H} 2$ type zinc finger $N b G I S$, AtGIS from Arabidopsis, and JcZFP8 from Jatropha curcas can also promote glandular trichome development in tobacco through gibberellic acid (GA) signaling (Liu et al. 2017, 2018, Shi et al. 2018). However, the molecular mechanism of glandular trichomes initiation and development in tobacco still remains largely unknown.

The homeodomain-leucine zipper (HD-Zip) transcription factor family is unique to the plant kingdom, and characterized by two indispensable conserved domains: the

Submitted 17 October 2019, last revision 2 January 2020, accepted 20 January 2020.

Abbreviations: DAI - days after infiltration; GA - gibberellic acid; MIXTA - myeloblastosis related transcription factor; qPCR quantitative PCR; SEM - scanning electron microscopy; SGT - short glandular trichome; TGT - tall glandular trichome; VIGS - virus induced gene silencing.

Acknowledgements: This study was supported by open foundation of the Molecular Genetics Key Laboratory of China Tobacco (No. MG[2014]) and the research programs CNTC-[2014]334-18, GZTC-[2011]-01, and CNTC-[2013]-159-JY04.

* Corresponding authors; e-mails: renxuel@126.com, xuying@scu.edu.cn 
homeodomain (HD) and the leucine zipper (Zip) domain (Schena and Davis 1992, Ariel et al. 2007). Based on the distinctive features of DNA-binding specificities, gene structures, additional common motifs, and physiological functions, the members of HD-Zip family can be divided into four groups, HD-Zip I to IV (Henriksson et al. 2005, Ariel et al. 2007). HD-Zip proteins play a wide variety of roles during plant growth and development, including lateral organ initiation (Otsuga et al. 2010), leaf cell expansion and endoreduplication (Hur et al. 2015), floral meristem regulation (Williams et al. 2005), and responses to biotic or abiotic stresses (Ariel et al. 2007, Brandt et al. 2014). The HD-Zip IV proteins have been demonstrated to play a pivotal role in trichome initiation in numerous plants. Glabrous 2 from Arabidopsis is the first homeodomain-leucine zipper IV gene identified, and this gene participates in trichome initiation (Cristina et al. 1996). Further studies on Arabidopsis demonstrate that other HD-Zip IV proteins, including protodermal factor 2 (Abe et al. 2003), homeodomain glabrous 2, homeodomain glabrous 11, and homeodomain glabrous 12 (Khosla et al. 2014) also participate in trichome formation and epidermal cell differentiation. Woolly (Wo) is a HDZip IV protein that affects trichome formation in tomato (Yang et al. 2011) and GL3 is a HD-Zip IV protein from cucumber. the GL3 spontaneous mutant cucumber plants, trichome-less (tril) and csgl3, show a completely glabrous phenotype (Pan et al. 2015, Zhao et al. 2015, Cui et al. 2016). Comparative transcriptomic analysis of the wild type and $g l 3$ mutant cucumber indicate that numerous transcription factors, which belonging to homeodomain, MADS, myeloblastosis, ethylene-responsive, and zinc finger family, are differently expressed (Zhao et al. 2015). In addition, Gossypium hirsutum homeodomain leucine zipper 1 (Walford et al. 2012) and Gossypium barbadense meristem layer 1 from cotton (Zhang et al. 2010), and outer cell layer 4 from maize (Vernoud et al. 2010), also belong to HD-Zip IV protein family and participate in trichome development.

The aim of this study was to investigate the expression pattern of $N b G L 3$, a HD-Zip IV family gene, in different tobacco organs and the expression of NbGL3 in response to plant hormones ethylene and auxin. In order to study the function of $N b G L 3$, we employed virus induced gene silencing (VIGS) system to silence the expression of NbGL3 and observed trichome development with scanning electron microscopy (SEM).

\section{Materials and methods}

Plants and growth conditions: Tobacco (Nicotiana benthamiana Domin) seedlings were cultured in a greenhouse under a $16-\mathrm{h}$ photoperiod, an irradiance of $100 \mu \mathrm{mol} \mathrm{m} \mathrm{m}^{-2} \mathrm{~s}^{-1}$, day/night temperatures of $23 / 19{ }^{\circ} \mathrm{C}$, and a relative humidity of $80 \%$. The seeds were germinated, the seedlings were grown until the second leaf could be observed, and then transplanted into the small basins. Then the seedlings with 4 - 5 true leaves (about $20 \mathrm{~d}$ after transplantation) were used for Agrobacterium infiltration.
The wild type tobacco plants about two months after transplantation which had entered flowering stage were used to collect samples (root, stem, mature leaf, young leaf, petiole, petal, sepal, fruit) for NbGL3 expression pattern analysis. Systemic leaves 20 days after infiltration (DAI) were used for RNA extraction and VIGS gene verification. The leaves one week after infiltration were collected for RNA extraction and downstream gene expressions.

Vector construction: For VIGS vector construction, a 660 bp fragment from the $N b G L 3$ coding sequence was amplified with specific primers: 5' - AAGGTTACCGAATTCTCTA GATCTTGTTAATTCAGGCCTCGC - 3' and 5' - TG TCTTCGGGACATGCCCGGGGTCGAACCATCTGG TAGTATC-3'. Linearized $p T R V 2$ vector was generated using the XbaI and SmaI restriction enzymes. The PCR product was ligated with linearized $p T R V 2$ vector using the ClonExpress II one step cloning kit (Vazyme, Nanjing, China). The reconstructed vector was transformed into competent Escherichia coli cells and verified by sequencing. The verified $\mathrm{p} T R V 2: N b G L 3$, empty $p T R V 2$, and $p T R V 1$ vectors were introduced into Agrobacterium tumefaciens strain GV3101 by heat shock method.

Agrobacterium infiltration: A. tumefaciens strains carrying TRV-mediated vectors were cultured overnight at $28{ }^{\circ} \mathrm{C}$ in the appropriate antibiotic selection medium. Then the cultures were spun down and resuspended in infiltration medium (10 mM MES, $10 \mathrm{mM} \mathrm{MgCl}_{2}$, $200 \mathrm{mM}$ acetosyringone) to an absorbance of 1 at $600 \mathrm{~nm}$. The resuspended cells were incubated at room temperature for $3 \mathrm{~h}$. Before infiltration, A. tumefaciens strain harboring $p T R V 1$ vector were mixed with that harboring $\mathrm{p} T R V 2: N b G L 3$ or $p T R V 2$ empty vector at a volume ratio of $1: 1$. The strain harboring $\mathrm{p} T R V 2: N b P D S$ was used as a positive control to assess the efficiency of VIGS system. $N$. benthamiana infiltration was performed as previously reported (Senthilkumar et al. 2014).

Plant hormone treatment: To examine the effect of plant hormones on NbGL3 expression, the tobacco plants (20 DAI) were sprayed with GA $(100 \mu \mathrm{M})$, 6-benzylaminopurine (6-BA; $50 \mu \mathrm{M})$, methyl jasmonate (MeJA; $100 \mu \mathrm{M})$, naphthaleneacetic acid (NAA; $100 \mu \mathrm{M})$, or 1-amino-1-cyclopropanecarboxylic acid (ACC; $1 \mu \mathrm{M}$ ), respectively. The control plants were sprayed with their corresponding mock solutions. The fourth leaf from the top at least from three plants at 3 and $6 \mathrm{~h}$ after treatment were harvested for RNA extraction.

Real-time quantitative PCR (qPCR) analysis: Total RNA was extracted using the plant total RNA isolation kit (Foregene, Chengdu, China). The cDNA was synthesized using PrimeScript ${ }^{\mathrm{TM}}$ RT reagent kit (Takara, Dalian, China) according to the manufacturer's instructions. The qPCR was performed on a $C F X 96^{\mathrm{TM}}$ real-time PCR system using SYBR Green Master Mix (Vazyme). The housekeeping gene $G A P D H$ was used as an internal reference. The primers used are listed in Table 1Suppl. 
Scanning electron microscopy: Systemic leaves, stems, and sepals from the plants 20 DAI with $p T R V 2: N b G L 3$ or $p T R V 2$ empty vector were used for SEM analysis. Samples treatment and SEM images were conducted as previously described (Chen et al. 2014).

\section{Results}

To determine the $N b G L 3$ function in glandular trichome development, we first investigated the expressions of $N b G L 3$ in different organs and developmental stages. Our results showed that $N b G L 3$ was expressed in all organs, and the highest expression occurred in mature leaves. Sepals and petals also possessed relatively high transcript abundance, while transcript abundance in root was quite low (Fig. $1 A$ ). The expression pattern of $N b G L 3$ was relatively consistent with the distribution of glandular trichomes, which indicated that $N b G L 3$ probably participated in glandular trichome development in tobacco.

Plant hormone plays an important role in trichome development. In order to study whether $N b G L 3$ controls the trichome initiation through hormone signaling, we first examined whether the expression of $N b G L 3$ in mature leaves could be induced by the application of plant hormones. Tobacco plants were sprayed with $100 \mu \mathrm{M} \mathrm{GA}$, $50 \mu \mathrm{M}$ 6-BA, $100 \mu \mathrm{M}$ MeJA, $100 \mu \mathrm{M}$ NAA, or $1 \mu \mathrm{M}$ ACC and the leaves were harvested at 3 and $6 \mathrm{~h}$ after hormone application. The real-time qPCR analysis was performed to examine the $N b G L 3$ transcript abundance. The expression of $N b G L 3$ was significantly higher in NAA and ACC treated plants than in their negative controls. However, GA, 6-BA, and MeJA treatment plants had no effect on $N b G L 3$ expression. These results suggest that NbGL3 expression could be induced by auxin and ethylene.

In order to explore the role of $N b G L 3$ in tobacco, we employed VIGS system to silence its expression. The Nicotiana benthamiana phytoene desaturase gene was used as a positive control to assess the efficiency of VIGS system. At 20 DAI, tobacco plants showed systematical bleaching phenotype, which indicated successful silencing Nicotiana benthamiana phytoene desaturase (Fig. 1 Suppl.). Real-time qPCR analysis performed on leaves, stems, and sepals collected from the infiltrated plants at 20 DAI further confirmed silencing Nicotiana benthamiana phytoene desaturase (Fig. 1 Suppl.). The transcription of $N b G L 3$ gene was significantly decreased in the $p T R V 2: N b G L 3$ infiltrated plants comparing with that infiltrated with the $p T R V 2$ empty vector (Fig. 1C). These results suggested that $N b G L 3$ gene was successfully silenced.

The expression of $N b G L 3$ was significantly reduced in $p T R V 2: N b G L 3$ infiltrated plant (Fig. 1C). To assess the effect of $N b G L 3$ on glandular trichome development, we checked the glandular trichome density on leaves, stems, and flower sepals from $p T R V 2: N b G L 3$ and $p T R V 2$ empty vector infiltrated plants. Compared to empty vector control plants, a decrease in glandular trichome density was observed in NbGL3 silenced plants (Figs $2 A$ and $3 A$ ). In order to determine whether $N b G L 3$ regulates glandular trichome initiation in tobacco, we counted the number of glandular trichomes in $p T R V 2: G L 3$ and $p T R V 2$ empty vector infiltrated plants. The results showed that trichome number per area was significantly decreased on $N b G L 3$ gene silenced leaves, stems, and sepals comparing with the negative control plants (Figs $2 B$ and $3 B$ ).

There are two kinds of glandular trichomes on $N$. benthamiana: short glandular trichomes (SGTs) and the tall glandular trichomes (TGTs) (Fig. 2 Suppl.). The SGTs
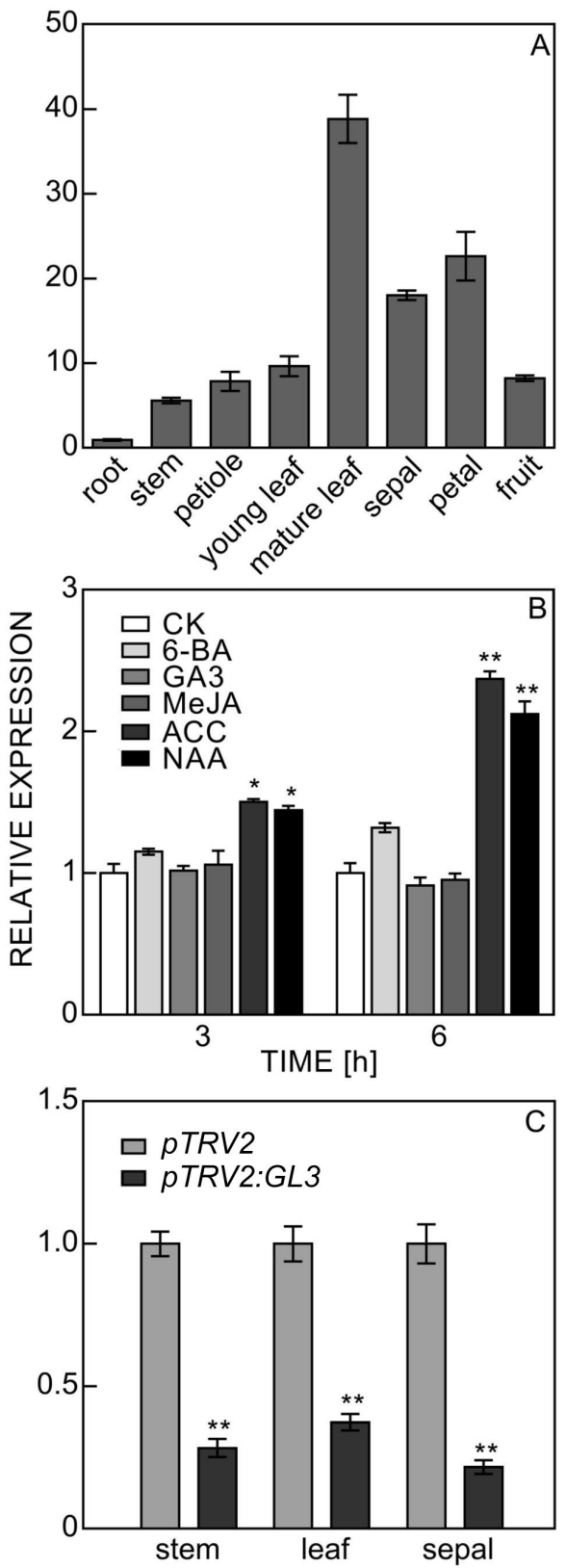

Fig. 1. Real-time quantitative PCR analysis of the $N b G L 3$ gene. $A$ - The expression pattern of the $N b G L 3$ gene in different organs. $B$ - Expression of the $N b G L 3$ gene in response to different plant hormones. $C$ - Expression of the NbGL3 gene in different organs treated with virus induced gene silencing. Expression in control plants was set to 1 . TRV2 - control plant treated with the TRV2 empty vector. Means \pm SEs, $n=3, *-P<0.05, * *-P<0.01$ (the Student's $t$-test). 


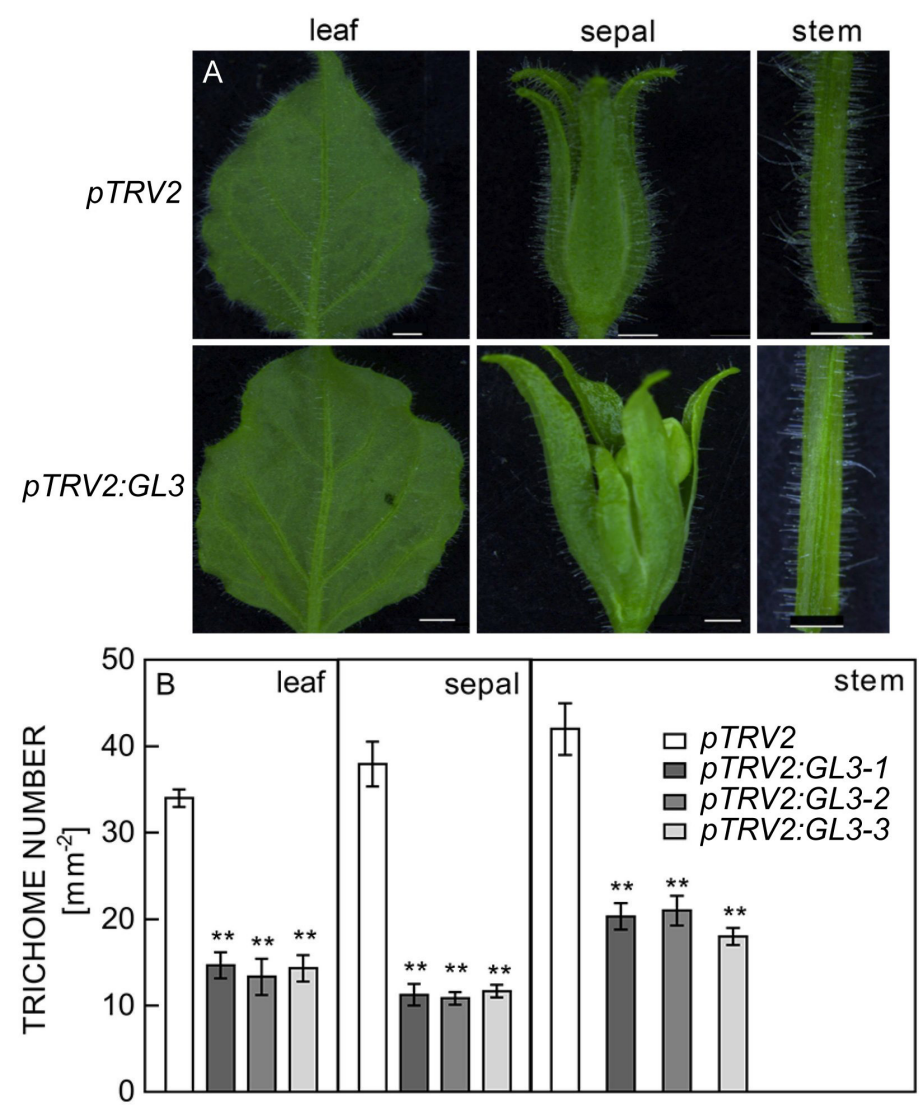

Fig. 2. Glandular trichomes on different organs. $A$ - Trichomes on different organs from NbGL3 silenced (pTRV2:GL3) and control (pTRV2) plants. $B$ - Glandular trichome density on leaves, sepals, and stems in $N b G L 3$ silenced and control (pTRV2) plants. Three TRV2:NbGL3 infiltrated plants were used for analysis. Means \pm SEs, $n=3,{ }^{*}-P<0.05$, ** $-P<0.01$ (the Student's $t$-test).

have a short unicellular stalk, on the top of which sits a head containing several nonchlorophyllous cells. The TGTs have a multicellular stalk and a head with a single or several chlorophyllous cells (Nielsen et al. 1991). NbGL3 silenced plant showed a reduction of glandular trichome density. To determine which kind of glandular trichome initiation was affected by $N b G L 3$, SEM was performed to observe the glandular trichome phenotypes in detail. SEM images showed that the ratio of TGTs and SGTs on different organs was different. Tobacco leaves and sepal edge showed a relatively higher amount of TGT, while sepal and stem had an opposite phenotype (Fig. 3A,B). The TGTs were not be observed on $N b G L 3$ silenced plant leaves, stems, and sepals. The SGTs density showed a mixed result, with an increased density on leaves and a decreased on sepals and stems. These results indicated that $N b G L 3$ could promote the long glandular trichomes initiation.

Silencing $N b G L 3$ in tobacco resulted in inhibition of long glandular trichomes; this results indicated that $N b G L 3$ might function in a similar way as its homologous genes in cucumber and tomato (Yang et al. 2011, Cui et al. 2016). In order to further confirm the function of $N b G L 3$ in tobacco, real-time qPCR analysis was conducted to examine the transcripts abundance of the genes whose homologs were differently expressed in tril and csgl3 mutant plant. As shown in Fig. 4, expression of all downstream genes were decreased in $N b G L 3$ silenced plants, and our results were consistent with previous reports (Yang et al. 2011, Cui et al. 2016, Wang et al. 2016).

\section{Discussion}

A key and unique feature of glandular trichomes is their ability to synthesize and secrete large amounts of specialized metabolites (Huchelmann et al. 2017). As such, they can be considered as "chemical factories", making them interesting targets for metabolic engineering (Schilmiller et al. 2010, Huchelmann et al. 2017). Elucidating the transcription network regulating glandular trichome development will provide great foundation for metabolic engineering targeting to the chemicals produced by glandular trichomes. Genes controlling trichome development have been extensively studied in Arabidopsis. However, according to the present knowledge, mechanisms controlling the multicellular glandular trichome development in tomato and tobacco is partially different from that of unicellular trichomes in Arabidopsis and cotton (Serna and Cathie 2006). GLABROUS1 (GL1) is a myeloblastosis gene required for trichome development in Arabidopsis (Marks and Feldmann 1989). However, overexpression of GL1 in tobacco could not induced trichome formation (Payne et al. 1999). Overexpression 

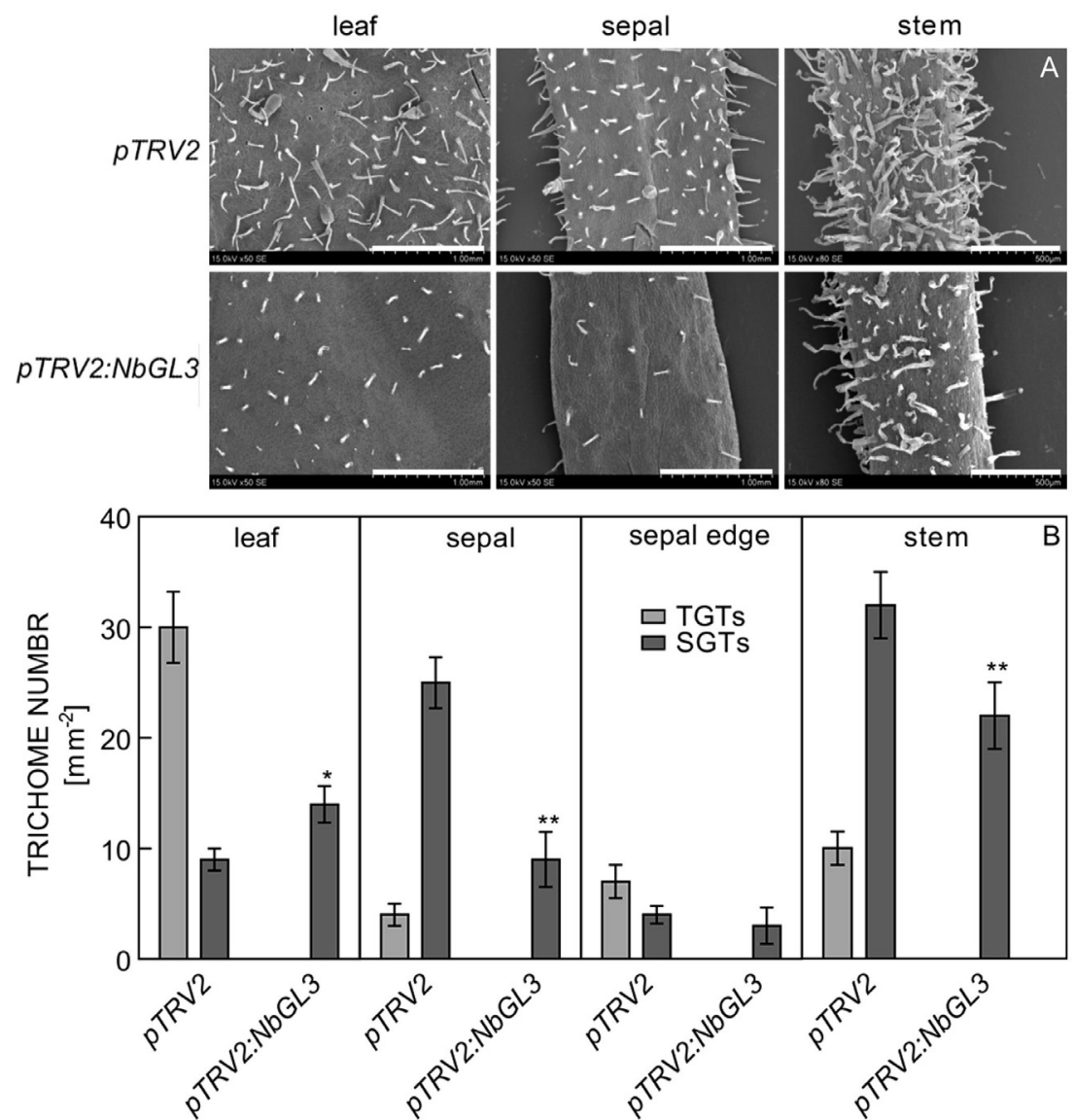

Fig. 3. Glandular trichomes analyzed by scanning electron microscopy. $A$ - Glandular trichomes on different organs from $N b G L 3$ silenced and control (pTRV2) plants. Scale bars: $1.00 \mathrm{~mm}$ (left and middle) and $500 \mu \mathrm{m}$ (right). B - Glandular trichome density on different organs from $N b G L 3$ silenced and control (pTRV2) plants. TGT - tall glandular trichome, SGT - short glandular trichome. Means \pm SEs, $n=3, *-P<0.05, * *-P<0.01$ (the Student's $t$-test).

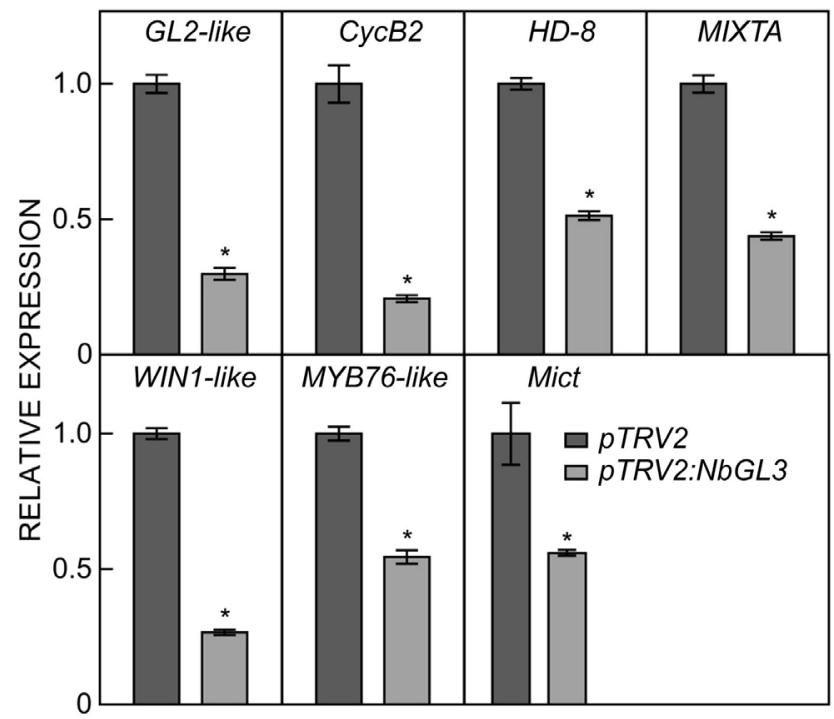

Fig. 4. Real-time quantitative PCR analysis of seven putative downstream genes of $N b G L 3$. Leaves from $N b G L 3$ silenced and control (pTRV2) plants were used for analysis. GL2-like - glabrous 2 like, CycB2 - cyclin B2, HD-8 - homeodomain 8, MIXTA - myeloblastosis related transcription factor, WIN1-like - wax inducer 1 like, MYB76-like - myeloblasosis 76-like, Mict - micro trichome. Means \pm SEs, $n=3, *-P<0.05$ (the Student's $t$-test). 
of MIXTA, a myeloblastosis related transcription factor controlling conical cell formation in snapdragon, could boost trichome initiation in tobacco (Payne et al. 1999) and A. majus (Glover et al. 1998). Whereas ectopic expression of MIXTA in Arabidopsis gll mutant plants failed to rescue the glabrous phenotype (Payne et al. 1999).

As described above, HD-Zip IV proteins seem to play an important roles in trichome development. In this study, we investigated the role of NbGL3, a HD-Zip IV protein, in $N$. benthamiana. The expression pattern of NbGL3 showed that $N b G L 3$ has a relatively higher transcription in leaves and sepals (Fig. 1A), this expression pattern was consistent with the TGTs distribution. In Arabidopsis, glabrous 2 promotes trichome initiation in shoots, but acts as a repressor in root hair development(Qing and Aoyama 2012). The low expression of NbGL3 in root indicated that $N b G L 3$ may not play an important role in root hair development in tobacco.

Plant hormones play an important role in trichome development in most plant. However, hormone regulation pathways are not conserved among different plants. In addition, different hormones may regulate trichome development through different transcription networks in the same species (Pattanaik et al. 2014). Previous reports showed that $A t G I S$ and NbGIS regulated glandular trichomes in tobacco through GA signaling pathway. In our study, expression of $N b G L 3$ only showed response to ethylene and auxin, and external gibberellin, cytokinin, and jasmonic acid methyl ester had no effect on $N b G L 3$ expression (Fig. 1B). These results indicated that $N b G L 3$ might regulate glandular trichome development in tobacco through ethylene and auxin signaling.

In tobacco plant, tall and short glandular trichome types have been identified (Nielsen et al. 1991). While the former have been extensively studied and match the classic picture of trichome function, the short trichomes have remained less characterized (Sallets et al. 2014). TGTs were completely disappeared on NbGL3 silenced plant surfaces (Fig. 3A,B). However, the changes of SGTs densities were more complex, with an increase on leaves and a decrease on sepals and stems (Fig. $3 B$ ). These results indicated that development of TGTs and SGTs might be controlled by different mechanisms and NbGL3 mainly functioned in TGTs initiation. Wo is a HD-Zip IV protein and regulates glandular trichomes development in tomato. Wo could induce the expression of a cell cycle protein coding gene, cyclin B2, and further physically interacted with cyclin B2 protein to regulate glandular trichome development (Yang et al. 2011, 2015). Transcriptomic analysis in cucumber csgl3 and tril mutant (Zhao et al. 2015, Cui et al. 2016, Wang et al. 2016), tomato wolly plant (Yang et al. 2011), and tobacco plant transformed with $W^{v}$ from tomato (Yang et al. 2015) identified a number of putative downstream genes. Several putative downstream genes were analyzed by real-time qPCR in the present study and our results were consistent with previous reports (Fig. 4). However, further studies have to be conducted to confirm if the NbGL3 protein could directly bind to the promoter region of the downstream genes to regulate their expression.

\section{References}

Abe, M., Katsumata, H., Komeda, Y., Takahashi, T.: Regulation of shoot epidermal cell differentiation by a pair of homeodomain proteins in Arabidopsis. - Development 130: 635, 2003.

Ariel, F.D., Manavella, P.A., Dezar, C.A., Chan, R.L.: The true story of the HD-Zip family. - Trends Plant Sci. 12: 419-426, 2007.

Brandt, R., Cabedo, M., Xie, Y., Wenkel, S.: Homeodomain leucine-zipper proteins and their role in synchronizing growth and development with the environment. - J. integr. Plant Biol. 56: 518-526, 2014.

Chen, C., Liu, M., Jiang, L., Liu, X., Zhao, J., Yan, S., Zhang, X.: Transcriptome profiling reveals roles of meristem regulators and polarity genes during fruit trichome development in cucumber (Cucumis sativus L.). - J. exp. Bot. 65: 4943-4958, 2014.

Cui, J.Y., Miao, H., Ding, L.H., Wehner, T.C., Liu, P.N., Wang, Y., $\mathrm{Gu}, \mathrm{X} . \mathrm{F} .:$ A New glabrous gene (csgl3) identified in trichome development in cucumber (Cucumis sativus L.). - PLoS ONE 11: e0148422, 2016.

Di Cristina, M., Sessa, G., Dolan, L., Linstead, P., Baima, S., Ruberti, I., Morelli, G..: The Arabidopsis Athb-10 (GLABRA2) is an HD-Zip protein required for regulation of root hair development. - Plant J. 10: 393-402, 1996.

Glover, B.J., Perez-Rodriguez, M., Martin, C.: Development of several epidermal cell types can be specified by the same MYB-related plant transcription factor. - Development 125: 3497, 1998.

Henriksson, E., Olsson, A.S., Johannesson, H., Johansson, H., Hanson, J., Engström, P., Söderman, E.: Homeodomain leucine zipper class I genes in Arabidopsis. Expression patterns and phylogenetic relationships. - Plant Physiol. 139: 509-518, 2005.

Huchelmann, A., Boutry, M., Hachez, C.: Plant glandular trichomes: natural cell factories of high biotechnological interest. - Plant Physiol. 175: 6-22, 2017.

Hur, Y.S., Um, J.H., Kim, S., Kim, K., Park, H.J., Lim, J.S., Lim, J.: Arabidopsis thaliana homeobox 12 (ATHB12), a homeodomain-leucine zipper protein, regulates leaf growth by promoting cell expansion and endoreduplication. - New Phytol. 205: 316-328, 2015.

Kang, J.H., Campos, M.L., Zemelisdurfee, S., Alhaddad, J.M., Jones, A.D., Telewski, F.W., Howe, G.A.: Molecular cloning of the tomato Hairless gene implicates actin dynamics in trichome-mediated defense and mechanical properties of stem tissue. - J. exp. Bot. 67: 5313-5324, 2016.

Kang, J.H., Mcroberts, J., Shi, F., Moreno, J.E., Jones, A.D., Howe, G.A.: The flavonoid biosynthetic enzyme chalcone isomerase modulates terpenoid production in glandular trichomes of tomato. - Plant Physiol. 164: 1161, 2014.

Khosla, A., Paper, J.M., Boehler, A.P., Bradley, A.M., Neumann, T.R., Schrick, K.: HD-Zip proteins GL2 and HDG11 have redundant functions in Arabidopsis trichomes, and GL2 activates a positive feedback loop via MYB23. - Plant Cell 26: 2184-2200, 2014.

Liu, Y., Liu, D., Hu, R., Hua, C., Ali, I., Zhang, A., Gan, Y.: AtGIS, a $\mathrm{C} 2 \mathrm{H} 2$ zinc-finger transcription factor from Arabidopsis regulates glandular trichome development through GA signaling in tobacco. - Biochem. biophys. Res. Commun. 483: 209-215, 2017.

Liu, Y., Liu, D., Khan, A. R., Liu, B., Wu, M., Huang, L., Gan, Y.: NbGIS regulates glandular trichome initiation through GA signaling in tobacco. - Plant mol. Biol. 98: 153-167, 2018.

Marks, M.D., Feldmann, K.A.: Trichome Ddvelopment in 
Arabidopsis thaliana. I. T-DNA tagging of the GLABROUS1 gene. - Plant Cell 1: 1043-1050, 1989.

Nielsen, M.T., Akers, C.P., Järlfors, U.E., Wagner, G.J., Berger, S.: comparative ultrastructural features of secreting and nonsecreting glandular trichomes of two genotypes of Nicotiana tabacum L. - Bot. Gaz. 152: 13-22, 1991.

Otsuga, D., Deguzman, B., Prigge, M.J., Drews, G.N., Clark, S.E.: REVOLUTA regulates meristem initiation at lateral positions. - Plant J. 25: 223-236, 2010.

Pan, Y., Bo, K., Cheng, Z., Weng, Y.: The loss-of-function GLABROUS 3 mutation in cucumber is due to LTRretrotransposon insertion in a class IV HD-ZIP transcription factor gene $C s G L 3$ that is epistatic over $C s G L 1$. - BMC Plant Biol. 15: 302, 2015.

Pattanaik, S., Patra, B., Singh, S.K., Yuan, L.: An overview of the gene regulatory network controlling trichome development in the model plant Arabidopsis. - Front. Plant Sci. 5: 259, 2014.

Payne, T., Clement, J., Arnold, D., Lloyd, A.: Heterologous $m y b$ genes distinct from $G L 1$ enhance trichome production when overexpressed in Nicotiana tabacum. - Development 126: 671-682, 1999.

Pesch, M., Hülskamp, M.: One, two, three models for trichome patterning in Arabidopsis. - Curr. Opin. Plant Biol. 12: 587592, 2009.

Qing, L., Aoyama, T.: Pathways for epidermal cell differentiation via the homeobox gene GLABRA2: update on the roles of the classic regulator F. - J. integr. Plant Biol. 54: 729-737, 2012.

Roda, A.L., Oldham, N.J., Svatos, A., Baldwin, I.T.: Allometric analysis of the induced flavonols on the leaf surface of wild tobacco (Nicotiana attenuata). - Phytochemistry 62: 527-536, 2003.

Sallets, A., Beyaert, M., Boutry, M., Champagne, A.: Comparative proteomics of short and tall glandular trichomes of Nicotiana tabacum reveals differential metabolic activities. - J. Proteome Res. 13: 3386-3396, 2014.

Schena, M., Davis, R.W.: HD-Zip proteins: members of an Arabidopsis homeodomain protein superfamily. - Proc. nat. Acad. Sci. USA 89: 3894-3898, 1992.

Schilmiller, A.L., Last, R.L., Pichersky, E.: Harnessing plant trichome biochemistry for the production of useful compounds. - Plant J. 54: 702-711, 2010.

Senthilkumar, M., Mysore, K.S.: Tobacco rattle virus-based virus-induced gene silencing in Nicotiana benthamiana. Natur. Protocols 9: 1549-1562, 2014.

Serna, L., Cathie, M. : Trichomes: different regulatory networks lead to convergent structures. - Trends Plant Sci. 11: 274-280, 2006.
Shi, X., Gu, Y., Dai, T., Wu, Y., Wu, P., Xu, Y., Chen, F. : Regulation of trichome development in tobacco by JcZFP8, a $\mathrm{C} 2 \mathrm{H} 2$ zinc finger protein gene from Jatropha curcas L. - Gene 658: 47-53, 2018.

Vernoud, V., Laigle, G., Rozier, F., Meeley, R. B., Perez, P., Rogowsky, P. M. : The HD-ZIP IV transcription factor OCL4 is necessary for trichome patterning and anther development in maize. - Plant J. Cell mol. Biol. 59: 883-894, 2010.

Wagner, G.J., Wang, E., Shepherd, R.W. : New approaches for studying and exploiting an old protuberance, the plant trichome. - Ann. Bot. 93: 3-11, 2004.

Walford, S.A., Wu, Y., Llewellyn, D.J., Dennis, E.S.: Epidermal cell differentiation in cotton mediated by the homeodomain leucine zipper gene, GhHD-1. - Plant J. 71: 464-478, 2012.

Wang, Y.L., Nie, J.T., Chen, H.M., Guo, C.L., Pan, J., He, H.L., Cai, R.: Identification and mapping of Tril, a homeodomainleucine zipper gene involved in multicellular trichome initiation in Cucumis sativus. - Theor. appl. Genet. 129: 305316, 2016.

Werker, E. : Trichome diversity and development. - Adv. bot. Res. 31: 1-35, 2000.

Williams, L., Grigg, S.P., Xie, M., Christensen, S., Fletcher, J.C. : Regulation of Arabidopsis shoot apical meristem and lateral organ formation by microRNA miR166g and its AtHD-ZIP target genes. - Development 132: 3657-3668, 2005.

Yan, A., Wu, M., Zhao, Y., Zhang, A., Gan, Y.: Involvement of $\mathrm{C} 2 \mathrm{H} 2$ zinc finger proteins in the regulation of epidermal cell fate determination in Arabidopsis. - J. integr. Plant Biol. 56: 1112-1117, 2014.

Yang, C., Gao, Y., Gao, S., Yu, G., Xiong, C., Chang, J., Ye, Z.: Transcriptome profile analysis of cell proliferation molecular processes during multicellular trichome formation induced by tomato Wov gene in tobacco. - BMC Genomics 16: 868, 2015.

Yang, C., Li, H., Zhang, J., Luo, Z., Gong, P., Zhang, C., Ye, $Z$.: A regulatory gene induces trichome formation and embryo lethality in tomato. - Proc. nat. Acad. Sci. USA 108: 1183611841, 2011

Zhang, F., Zuo, K., Zhang, J., Liu, X., Zhang, L., Sun, X., Tang, K.: An L1 box binding protein, GbML1, interacts with GbMYB25 to control cotton fibre development. - J. exp. Bot. 61: 3599-3613, 2010.

Zhao, J.L., Wang, Y.L., Yao, D.Q., Zhu, W.Y., Chen, L., He, H.L., Cai, R.: Transcriptome profiling of trichome-less reveals genes associated with multicellular trichome development in Cucumis sativus. - Mol. Genet. Genomics 290: 2007-2018, 2015. 